\title{
Postoperative Outcome in Non-Preterm Infants Under One Year Old in Non-Cardiac Surgery
}

\section{Claudine Kumba*}

Department of Pediatric and Obstetric Anesthesia and Critical Care, Hôpital Universitaire Necker Enfants Malades, Assistance Publique Hôpitaux de Paris, APHP, Université de Paris, Paris, France

*Corresponding Author: Claudine Kumba, Department of Pediatric and Obstetric Anesthesia and Critical Care, Hôpital Universitaire Necker Enfants Malades, Assistance Publique Hôpitaux de Paris, APHP, Université de Paris, Paris, France.
Received: May 10, 2021

Published: July 05, 2021

(C) All rights are reserved by Claudine Kumba.

\section{Abstract}

Background: An observational study conducted earlier to determine predictors of postoperative outcome in non-cardiac surgical pediatric patients showed that factors which influenced postoperative evolution were multiple. These included American Society of Anesthesiologists (ASA) score, transfusion, age, emergency surgery, and surgery.

Objectives: To describe in details outcomes in non-preterm children under one year old included in the initial study.

Methods: Secondary analysis of the initial retrospective observational study in 594 patients with a mean age of $90.86 \pm 71.80$ months.

The Ethics Committee approved the study under the registration number 2017-CK-5-R1.

Results: There were 97 non-preterm children included with a mean age of $4.44 \pm 3.49$ months.

Mean weight was $5.13 \pm 2.74$ kilograms. There were 48 abdominal surgical patients (49.49\%), 48 neurosurgical patients (49.49\%) and 1 orthopedic surgery patient (1.03\%). 30 patients had intra-operative and or postoperative complications (organ failure or sepsis) (30.93\%). The most common intra-operative complication was hemorrhagic shock (5.16\%); the most affected system in the postoperative period was the respiratory system in terms of organ failure and pulmonary sepsis with an overall rate of $12.38 \%$; the most common postoperative infection was septicemia (7.22\%). The rate of postoperative renal failure was $1.03 \%$. There were 5 inhospital deaths (5.16\%) and all were ASA III, IV and V patients managed on an emergency basis.

Conclusion: In this cohort of 97 non-preterm infants under one year old, the rate of patients with intra-operative and or postoperative complications was 30.93\%. Patients with fatal outcome had an ASA score III or more and were managed on an emergency basis.

It is time to reconsider integrating goal directed therapies in intra-operative patient management to improve postoperative outcome.

Keywords: Children Under One Year Old; Outcome; Non-Cardiac Surgery

\section{Introduction}

An observational study conducted earlier to determine predictors of postoperative outcome in non-cardiac surgical pediatric patients concluded that predictors of postoperative evolution were multiple [1]. These included American Society of Anesthesiologists (ASA) status, transfusion, age, emergency surgery and surgery. Postoperative outcome in this observational trial was defined as

Citation: Claudine Kumba., et al. "Postoperative Outcome in Non-Preterm Infants Under One Year Old in Non-Cardiac Surgery". Acta Scientific Paediatrics 4.8 (2021): 11-23. 
intra-operative and postoperative complications (organ failure and sepsis), re-surgery, mortality, length of stay in the intensive care unit (LOSICU), length of stay in hospital (LOS), total length of stay in hospital, TLOS (LOSICU+LOS) and length of mechanical ventilation (LMV).

\section{Objective of the Study}

The study presented in this article had the objective to describe these outcomes in children under one year old in details.

\section{Methods}

Description of intra-operative and postoperative outcomes in children less than one year old included in the initial cohort of 594 patients aged $90.86 \pm 71.80$ months [1] .

The study was declared to the CNIL, National Commission for Computer Science and Liberties on 21 February 2017 under the registration number 2028257 v0. The Ethics Committee of Necker approved the study on 21 March 2017 under the registration number 2017-CK-5-R1. Patients were included retrospectively from 1 January 2014 to 17 May 2017.

Inclusion criteria were children aged less than one year and older than 37 weeks.

Exclusion criteria were children aged less than 37 weeks and older than one year.

Statistics were analyzed with XLSTAT 2020.4.1. software.

Continuous variables were described in means \pm standard deviation or medians with interquartile ranges. Categorial variables were described in proportions. Categoric variables were compared with Fischer's exact test. A p-value of less than 0.05 was considered significant.

\section{Results}

General characteristics are illustrated in table 1.

There were 97 children included with a mean age of $4.44 \pm 3.49$ months. Mean weight was $5.13 \pm 2.74$ kilograms. There were 48 abdominal surgical patients (49.49\%), 48 neurosurgical patients (49.49\%) and 1 orthopedic surgical patient (1.03\%). 57 patients had elective surgery (58.76\%) and 40 had emergency surgery (41.24\%). 11 patients had re-surgery (11.34\%). 30 patients had in-

\begin{tabular}{|c|c|}
\hline Characteristics & $\mathbf{N}=97$ \\
\hline Mean age in months & $4.44 \pm 3.49$ \\
\hline Mean weight in kilograms & $5.13 \pm 2.74$ \\
\hline Abdominal surgery n (\%) & $48(49.49)$ \\
\hline Neurosurgery n (\%) & $48(49.49)$ \\
\hline Orthopedic surgery n (\%) & $1(1.03)$ \\
\hline Elective surgery n (\%) & $57(58.76)$ \\
\hline Emergency surgery n (\%) & $40(41.24)$ \\
\hline Re-surgery n (\%) & $11(11.34)$ \\
\hline $\begin{array}{l}\text { Patients with intra-operative and or post- } \\
\text { operative complications (organ failure or } \\
\text { sepsis) n (\%) }\end{array}$ & $30(30.93)$ \\
\hline Intra-operative hemorrhagic shock n (\%) & $5(5.16)$ \\
\hline Intra-operative cardiac arrest n (\%) & $1(1.03)$ \\
\hline $\begin{array}{l}\text { Intra-operative bronchospasm or laryngo- } \\
\text { spasm n (\%) }\end{array}$ & $1(1.03)$ \\
\hline Postoperative neurologic failure n (\%) & $2(2.06)$ \\
\hline $\begin{array}{l}\text { Postoperative cardio-circulatory failure n } \\
(\%)\end{array}$ & $5(5.16)$ \\
\hline Postoperative respiratory failure n (\%) & $6(6.19)$ \\
\hline Postoperative renal failure n (\%) & $1(1.03)$ \\
\hline Postoperative miscellaneous n (\%) & $1(1.03)$ \\
\hline Postoperative multi-organ failure n (\%) & $5(5.16)$ \\
\hline Postoperative hemorrhagic shock n (\%) & $1(1.03)$ \\
\hline Postoperative pulmonary sepsis n (\%) & $6(6.19)$ \\
\hline Postoperative abdominal sepsis n (\%) & $6(6.19)$ \\
\hline Postoperative neuro-meningeal sepsis n (\%) & $2(2.06)$ \\
\hline Postoperative septicemia n (\%) & $7(7.22)$ \\
\hline Postoperative multi-organ sepsis n (\%) & $1(1.03)$ \\
\hline In hospital Mortality n (\%) & $5(5.16)$ \\
\hline Transfusion n (\%) & $67(69.07)$ \\
\hline $\begin{array}{l}\text { Mean preoperative hemoglobin levels } \pm \text { stan- } \\
\text { dard deviation } \mathrm{g} / \mathrm{dL}\end{array}$ & $12.07 \pm 3.09$ \\
\hline $\begin{array}{l}\text { Mean postoperative hemoglobin levels } \pm \\
\text { standard deviation } \mathrm{g} / \mathrm{dL}\end{array}$ & $12.40 \pm 2.54$ \\
\hline ASA I n (\%) & $31(31.96)$ \\
\hline ASA II n (\%) & $23(23.71)$ \\
\hline ASA III n (\%) & $26(26.80)$ \\
\hline ASA IV n (\%) & $15(15.46)$ \\
\hline ASA V n (\%) & $2(2.06)$ \\
\hline $\begin{array}{l}\text { Median length of intensive care unit stay in } \\
\text { days }\end{array}$ & $4[3-16]$ \\
\hline
\end{tabular}

Citation: Claudine Kumba., et al. "Postoperative Outcome in Non-Preterm Infants Under One Year Old in Non-Cardiac Surgery". Acta Scientific Paediatrics 4.8 (2021): 11-23. 


\begin{tabular}{|l|c|}
\hline Median length of hospital stay in days & 4 [1-18] \\
\hline Median total length of hospital stay in days & $12[4-34]$ \\
\hline $\begin{array}{l}\text { Median length of mechanical ventilation } \\
\text { (invasive or non-invasive) in days }\end{array}$ & $0[0-3]$ \\
\hline
\end{tabular}

Table 1: General characteristics.

tra-operative and or postoperative complications (organ failure or sepsis) (30.93\%). 5 patients had intra-operative hemorrhagic shock (5.16\%), 1 patient had an intra-operative cardiac arrest (1.03\%) and 1 patient had an intra-operative bronchospasm/laryngospasm (1.03\%). 2 patients had postoperative neurologic failure $(2.06 \%)$, 5 patients had postoperative cardio-circulatory failure $(5.16 \%), 6$ patients had postoperative respiratory failure (6.19\%), 1 patient had postoperative renal failure $(1.03 \%), 5$ patients had postoperative multi-organ failure (5.15\%), and 1 patient had postoperative hemorrhagic shock (1.03\%). 6 patients had postoperative pulmonary sepsis (6.19\%), 6 patients had postoperative abdominal sepsis (6.19\%), 2 patients had postoperative neuro-meningeal sepsis (2.6\%), 7 patients had postoperative septicemia (7.22\%) and 1 patient had postoperative multi-organ sepsis (1.03\%). 67 patients received transfusion intra-operatively (69.07\%). There were 5 inhospital deaths (5.16\%) and all were ASA III or more and were managed on an emergency basis. Among the deceased patients 1 had a liver transplantation, 1 had an intestinal resection, 1 had a laparotomy for volvulus, 1 had a cerebral aneurysm embolization and 1 had an extradural hematoma drainage (See table 2).

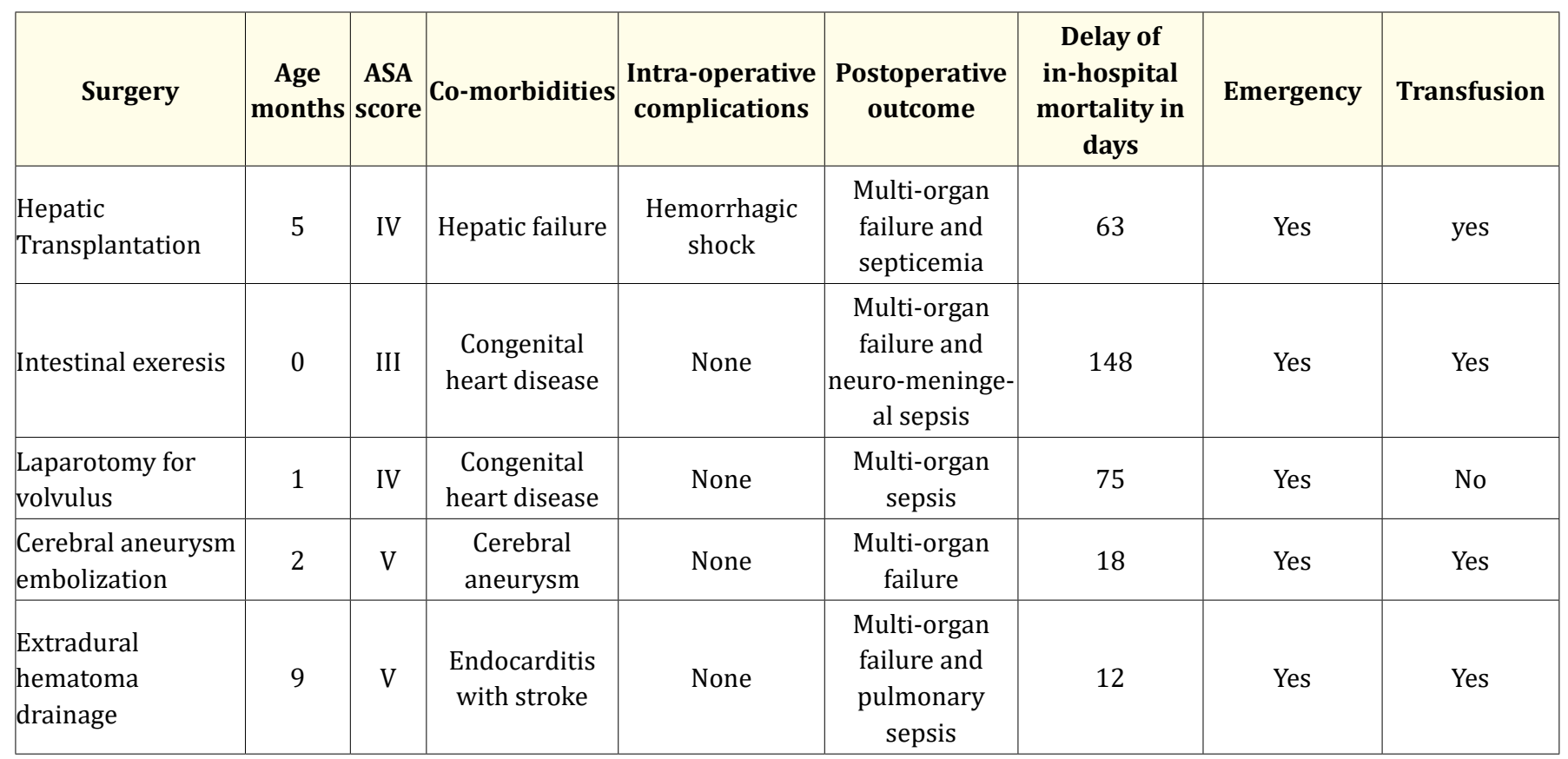

Table 2: Patients with fatal outcome.

There were 31 ASA I (31.96\%), 23 ASA II (23.71\%), 26 ASA III (26.80\%), 15 ASA IV (15.46\%) and 2 ASA V patients (2.06\%) (Table 3).

Median length of postoperative intensive care unit stay (LOSICU) was 4 days [3 - 16], median length of postoperative hospital stay (LOS) was 4 days [1 - 18], median total length of postoperative hospital stay, TLOS (LOSICU+LOS) was 12 days [4 - 34] and median length of postoperative mechanical invasive or non-invasive mechanical ventilation (LMV) was 0 days [0 - 3].

The majority of the patients $(69.07 \%)$ were transfused intraoperatively.

Mean preoperative hemoglobin levels were $12.07 \pm 3.09 \mathrm{~g} / \mathrm{dL}$ and mean postoperative hemoglobin levels were $12.40 \pm 2.54 \mathrm{~g} /$ dL. 


\begin{tabular}{|l|c|}
\hline \multicolumn{1}{|c|}{ Surgery } & $\begin{array}{c}\text { Number of } \\
\text { patients }\end{array}$ \\
\hline Ano-rectal malformation & 3 \\
\hline Neuroblastoma & 2 \\
\hline Hepatic transplantation & 7 \\
\hline Intestinal exeresis & 11 \\
\hline Hepatic tumor & 1 \\
\hline Nissen Gastrostomy & 1 \\
\hline Conjoined twin separation & 2 \\
\hline Kasai & 3 \\
\hline Mediastinal ganglioneuroma & 1 \\
\hline Esophageal atresia & 5 \\
\hline Pelvic tumor & 2 \\
\hline Laparotomy for volvulus & 4 \\
\hline Exploratory laparotomy & 1 \\
\hline Omphalocele & 5 \\
\hline Peritoneal or external & 3 \\
\hline ventriculostomy & 39 \\
\hline Craniosynostosis & 1 \\
\hline Cerebral aneurysm or arterio-venous \\
malformation embolization & 2 \\
\hline Central venous catheter & 1 \\
\hline Attached/Fixed spinal cord & 1 \\
\hline Intra-cerebral tumor exeresis & \\
\hline Extra-dural hematoma drainage & 1 \\
\hline Limb Tumor exeresis & 1 \\
\hline Total & 1 \\
\hline
\end{tabular}

Table 3: Surgery.

Table 3 illustrates types of surgery, the most common surgical intervention was craniosynostosis (39 patients; 40\%), followed by intestinal resection (11 patients; $11 \%$ )), hepatic transplantation (7 patients; $7 \%$ ), esophageal atresia (5 patients; $5 \%$ ), omphalocele (5 patients; $5 \%$ ) and laparotomy for volvulus (4 patients, $4 \%$ ).

Table 4 illustrates outcomes per surgery and table 5 illustrates p-values for complications per surgery.

\section{Discussion}

The most common intra-operative complication was hemorrhagic shock (5.16\%), followed by broncho-laryngo spasm

(1.03\%) and cardiac arrest (1.03\%). Among the most common interventions described in this manuscript, craniosynostosis and liver transplantation were among the most hemorrhagic interventions this explains the rate of intra-operative hemorrhagic shock. Transfusion rate was $69.07 \%$ in this study. A previous study in craniosynostosis has reported a transfusion rate of $100 \%$ which was reduced to $22.7 \%$ after an implementation program with aim to reduce transfusion [2] and another study reported a transfusion rate of $66 \%$ [3]. A study in liver transplantation in children revealed a massive transfusion rate of $55 \%$ [4].

The rate of intra-operative cardiac arrest was higher than that reported in a study of infants aged less than 60 weeks of postmenstrual age which showed a rate of $0.12 \%$ [5]. Nevertheless, this same study revealed that intra-operative critical events were present in $35.3 \%$ of the patients and the most common concerned the cardiovascular instability and hypoxemia [5]. In our study the rate of intra-operative critical events was $7.22 \%$ which included hemorrhagic shock, broncho-laryngospasm and cardiac arrest. The Nectarine study reported an intra-operative serious event rate of $35.3 \%$ and a 30 days morbidity rate of $16.3 \%$ with respiratory, surgical and cardiovascular complications as common events keeping in mind that this study included pre-terms and term patients up-to 60 weeks postmenstrual age [5]. Our study included term infants and pre-terms were not included.

In our study the rate of patients with intra-operative and or postoperative complications was $30.93 \%$. The respiratory system was the most affected in the postoperative period with overall complication rate of $12.38 \%$ which included respiratory failure (6.19\%) and pulmonary sepsis (6.19\%). According to 2 previous studies, postoperative pneumonia rate varied between $<1 \%$ to $1.2 \%[3,6,7]$. Re-surgery was present in $11.34 \%$ of the patients in our study. Previous studies have reported a re-operation rate between 2 and 5\% [3,8]. Postoperative cardio-circulatory failure $(5.16 \%)$ and multi-organ failure $(5.16 \%)$ were the second most frequent postoperative systemic failures followed by neurologic dysfunction which included neurologic failure (2.06\%) and neuromeningeal sepsis $(2.06 \%)$. According to a previous narrative review, the incidence multiple organ dysfunction in pediatric intensive care unit (PICU) varies from 6 to $57 \%$ according to the studies [9]. Mortality rates from multi-organ dysfunction varies from $<5 \%$ to $>80 \%$ depending on the number of organ failure [9]. The most 


\begin{tabular}{|c|c|c|c|c|c|c|c|c|c|c|c|c|c|c|c|c|c|c|c|}
\hline & 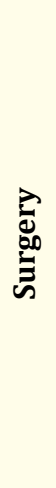 & 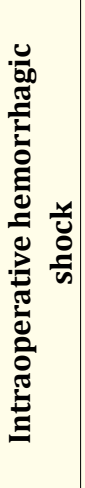 & 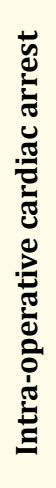 & 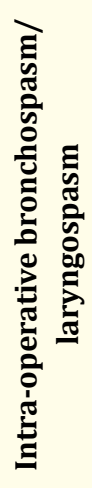 & 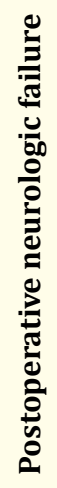 & 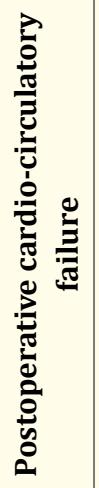 & 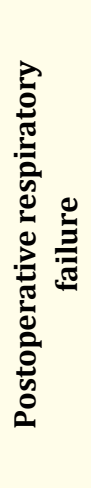 & 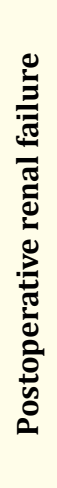 & 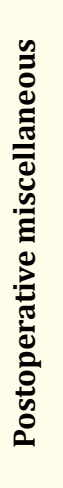 & 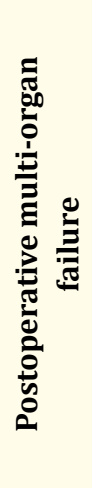 & 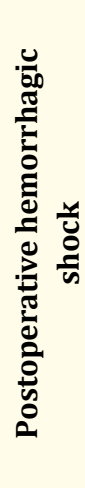 & 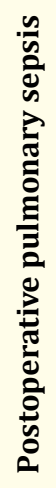 & 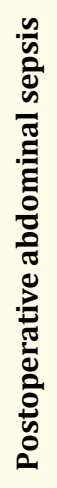 & 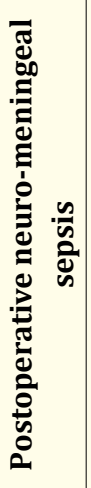 & 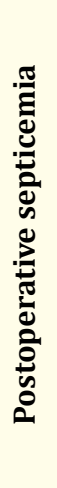 & 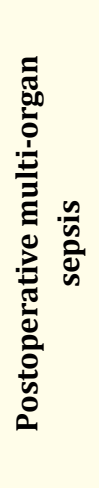 & 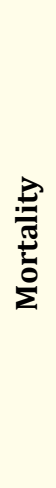 & 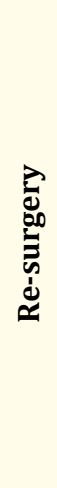 & 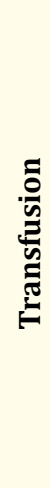 \\
\hline 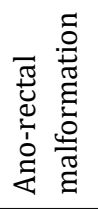 & $m$ & 0 & 0 & 0 & 0 & 0 & 0 & 0 & 0 & 0 & 0 & 0 & 0 & 0 & 0 & 0 & 0 & 0 & $N$ \\
\hline 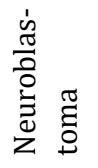 & $\sim$ & -1 & 0 & 0 & 0 & $\sim$ & 0 & 0 & 0 & 0 & 0 & 0 & 0 & 0 & 0 & 0 & 0 & 0 & $N$ \\
\hline 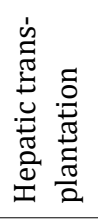 & 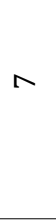 & $N$ & 0 & 0 & $r$ & $r$ & 0 & 0 & 0 & - & 0 & 0 & $N$ & 0 & $m$ & 0 & $r$ & $N$ & $\wedge$ \\
\hline 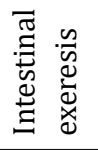 & $\exists$ & - & 0 & 0 & 0 & $r$ & $N$ & 0 & 0 & - & 0 & - & - & -1 & $r$ & 0 & $r$ & $N$ & $a$ \\
\hline 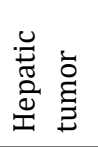 & - & 0 & 0 & 0 & 0 & 0 & 0 & 0 & 0 & 0 & 0 & 0 & 0 & 0 & 0 & 0 & 0 & 0 & - \\
\hline 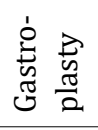 & $-r$ & 0 & 0 & 0 & 0 & 0 & $r$ & 0 & 0 & 0 & 0 & - & 0 & 0 & 0 & 0 & 0 & $\neg$ & - \\
\hline 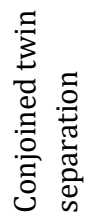 & $N$ & 0 & 0 & 0 & 0 & 0 & 0 & 0 & $\neg$ & 0 & 0 & $-r$ & $-r$ & 0 & 0 & 0 & 0 & -1 & $N$ \\
\hline
\end{tabular}




\begin{tabular}{|c|c|c|c|c|c|c|c|c|c|c|c|c|c|c|c|c|c|c|c|}
\hline $\begin{array}{l}\overline{\widetilde{\pi}} \\
\tilde{\widetilde{U}} \\
\tilde{\widetilde{y}}\end{array}$ & $m$ & 0 & 0 & 0 & 0 & 0 & 0 & 0 & 0 & 0 & 0 & 0 & $\rightarrow$ & 0 & 0 & 0 & 0 & 0 & $m$ \\
\hline 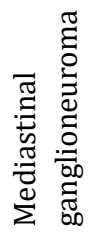 & $\rightarrow$ & 0 & 0 & 0 & 0 & - & 0 & 0 & 0 & 0 & 0 & 0 & 0 & 0 & -1 & 0 & 0 & $r$ & $r$ \\
\hline 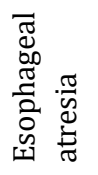 & $\llcorner\cap$ & 0 & 0 & 0 & 0 & 0 & $r$ & 0 & 0 & $r$ & 0 & $\neg$ & $\neg$ & 0 & 0 & 0 & 0 & 0 & - \\
\hline 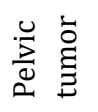 & $N$ & 0 & 0 & 0 & 0 & 0 & 0 & 0 & 0 & 0 & 0 & 0 & 0 & 0 & 0 & 0 & 0 & 0 & 0 \\
\hline 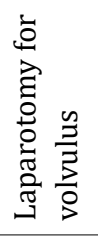 & $\sigma$ & 0 & 0 & 0 & 0 & 0 & 0 & 0 & 0 & 0 & 0 & 0 & 0 & 0 & $r$ & $\neg$ & $r$ & $r$ & 0 \\
\hline 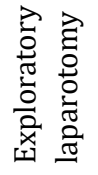 & $r$ & 0 & 0 & 0 & 0 & 0 & 0 & 0 & 0 & 0 & 0 & 0 & 0 & 0 & 0 & 0 & 0 & 0 & 0 \\
\hline 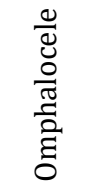 & \llcorner & 0 & 0 & 0 & 0 & 0 & 0 & $r$ & 0 & 0 & 0 & 0 & 0 & 0 & $r$ & 0 & 0 & 0 & 0 \\
\hline 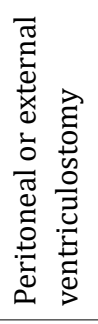 & $m$ & 0 & 0 & 0 & 0 & 0 & 0 & 0 & 0 & 0 & 0 & 0 & 0 & 0 & 0 & 0 & 0 & $\rightarrow$ & $-r$ \\
\hline 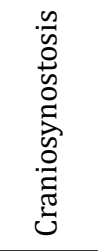 & & - & 0 & - & $r$ & 0 & $r$ & 0 & 0 & 0 & $r$ & 0 & 0 & $-r$ & 0 & 0 & 0 & $\rightarrow$ & $\vec{m}$ \\
\hline
\end{tabular}




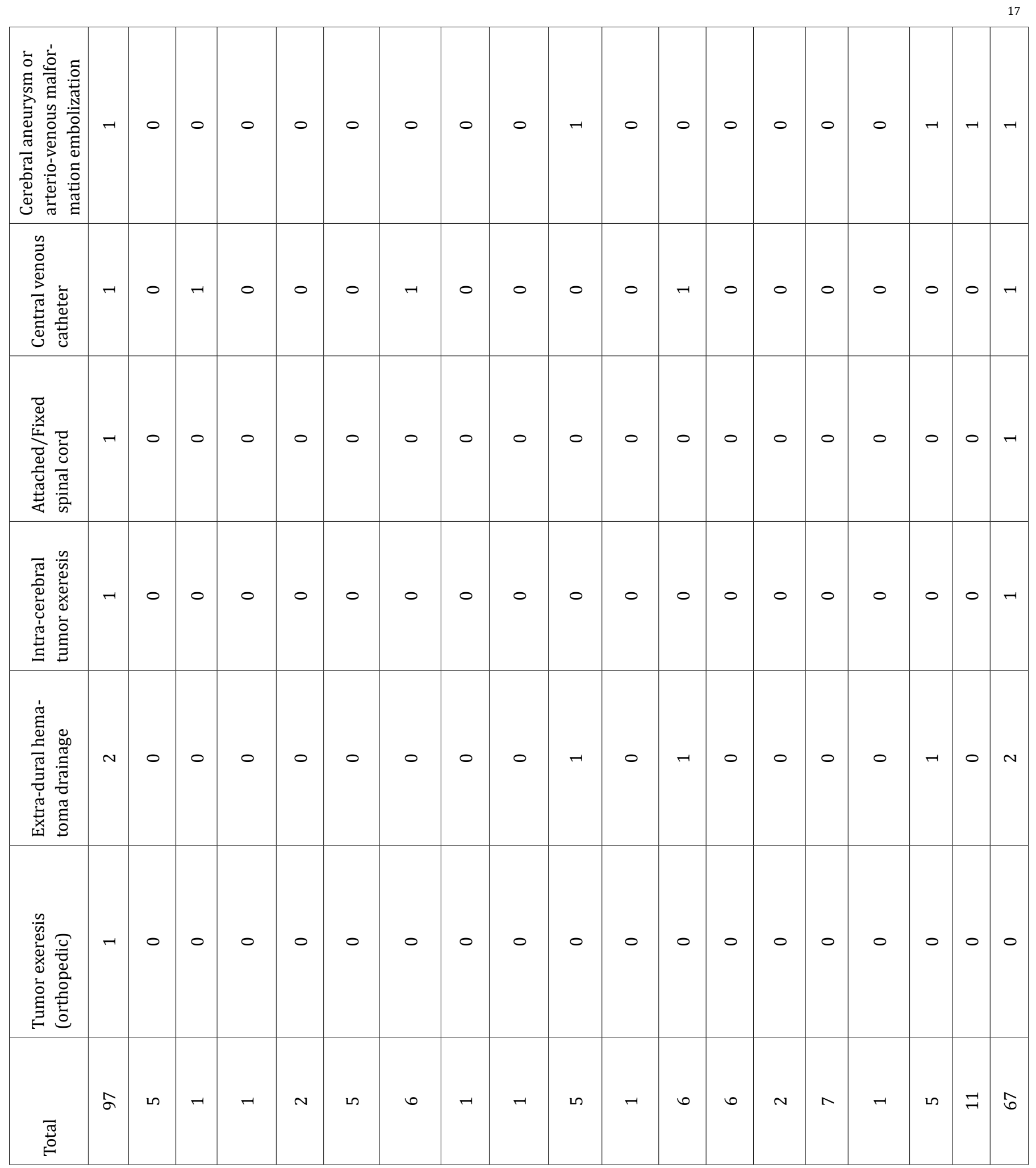

Table 4: Outcome per surgery. 


\begin{tabular}{|c|c|c|c|c|c|c|c|c|c|c|c|c|c|c|c|c|c|c|c|}
\hline & 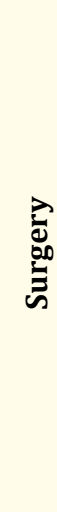 & 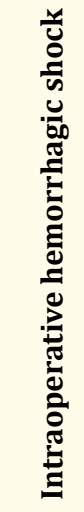 & 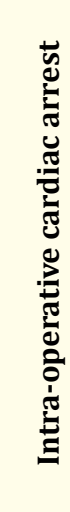 & 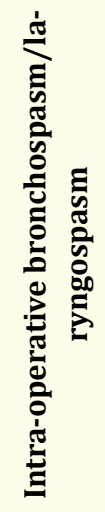 & 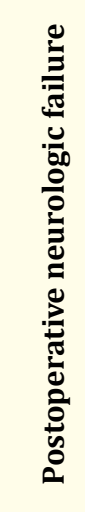 & 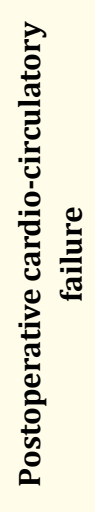 & 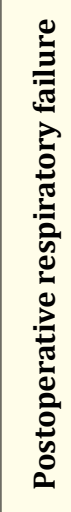 & 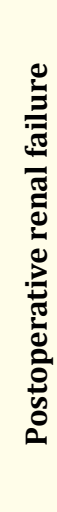 & 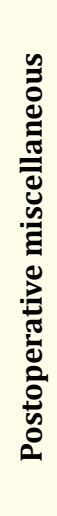 & 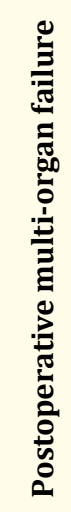 & 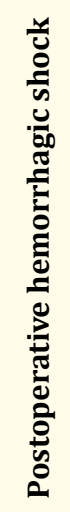 & 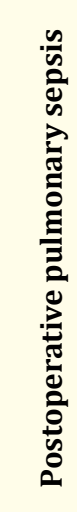 & 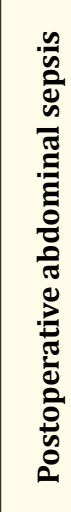 & 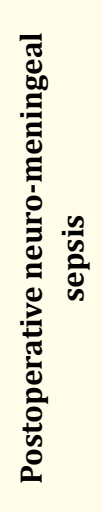 & 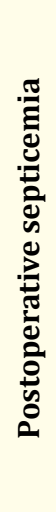 & 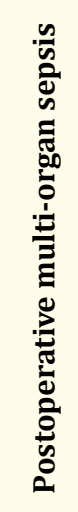 & 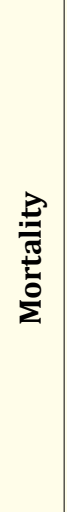 & 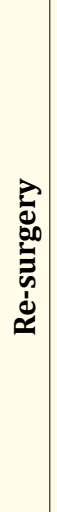 & 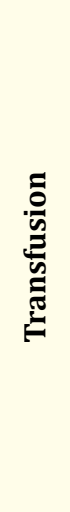 \\
\hline 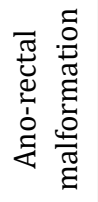 & $\begin{array}{l}\text { L } \\
0 \\
0\end{array}$ & $r$ & $r$ & $r$ & -1 & $r$ & -1 & -1 & 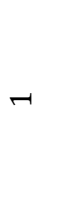 & $r$ & - & $r$ & $r$ & $r$ & $r$ & - & $r$ & $r$ & $\begin{array}{l}\tilde{0} \\
0\end{array}$ \\
\hline 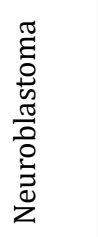 & $\begin{array}{l}\stackrel{P}{0} \\
0\end{array}$ & $\stackrel{+}{\vec{\sigma}}$ & $\neg$ & $r$ & $\neg$ & on & $r$ & $r$ & $r$ & $r$ & - & $r$ & $-r$ & $\neg$ & $-r$ & - & - & -1 & - \\
\hline 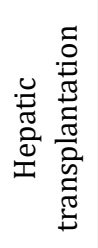 & $\begin{array}{l}\circ \\
\dot{0}\end{array}$ & $\begin{array}{l}\stackrel{0}{0} \\
\stackrel{-}{0}\end{array}$ & $r$ & $r$ & $\stackrel{\vec{N}}{0}$ & fे & $r$ & -1 & $\neg$ & f̊ & $\neg$ & $r$ & $\stackrel{\text { nn }}{\stackrel{0}{0}}$ & $\neg$ & $\begin{array}{l}\dot{J} \\
0 \\
0\end{array}$ & $r$ & $\stackrel{\text { Lִ }}{0}$ & $\begin{array}{l}\widetilde{U} \\
0\end{array}$ & \begin{tabular}{l} 
ळ \\
\hdashline
\end{tabular} \\
\hline 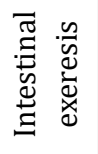 & $\underset{J}{J}$ & $\begin{array}{l}\text { ก? } \\
\text { ? }\end{array}$ & $\neg$ & - & $\neg$ & นn & 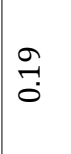 & $r$ & $r$ & חొ & $r$ & $\stackrel{0}{\circ}$ & $\stackrel{0}{0}$ & $\ddot{n}$ & $\rightarrow$ & - & ᄂ? & $\hat{o}$ & - \\
\hline 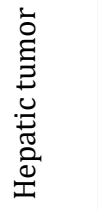 & $r$ & $\rightarrow$ & - & $\neg$ & - & -1 & - & -1 & $-r$ & $r$ & - & $\neg$ & $r$ & -1 & $r$ & - & $r$ & $r$ & $\begin{array}{l}\text { oे } \\
\text { ț } \\
0\end{array}$ \\
\hline 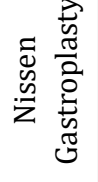 & $0^{\circ}$ & - & $r$ & - & $r$ & -1 & సै & -1 & -1 & $r$ & $r$ & ㄱ. & $r$ & -1 & -1 & - & $\neg$ & ֶָ. & $r$ \\
\hline
\end{tabular}









\begin{tabular}{|c|c|c|c|c|c|c|c|c|c|c|c|c|c|c|c|c|c|c|c|}
\hline 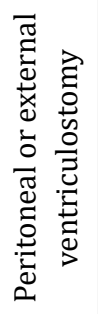 & $\hat{o}$ & $r$ & - & - & $r$ & -1 & - & - & - & $\neg$ & -1 & - & $\rightarrow$ & -1 & - & $\neg$ & $r$ & $\tilde{o}$ & - \\
\hline 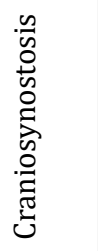 & $\begin{array}{l}\stackrel{0}{0} \\
\stackrel{0}{0}\end{array}$ & $\stackrel{0}{0}$ & - & $\stackrel{m}{0}$ & $r$ & ". & $\stackrel{0}{0}$ & $r$ & - & "ే. & $\ddot{0}$ & ָ̃. & $\tilde{o}$ & -1 & "). & $\rightarrow$ & ָ̃ & $\overrightarrow{0}$ & ¿- \\
\hline 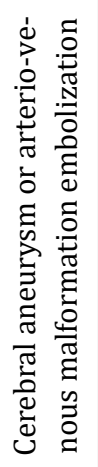 & $\stackrel{+}{0}$ & $r$ & $r$ & - & $-r$ & -1 & - & - & - & 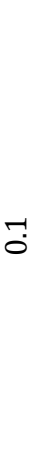 & $r$ & $\rightarrow$ & -1 & $r$ & $r$ & $r$ & $\stackrel{-}{0}$ & Ñ & - \\
\hline 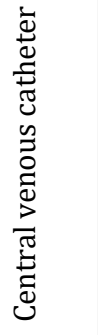 & ț. & - & $\begin{array}{l}\tilde{O} \\
\stackrel{0}{\circ}\end{array}$ & - & $r$ & -1 & - & - & - & - & 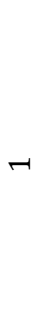 & - & $\rightarrow$ & - & $r$ & - & $\rightarrow$ & -1 & - \\
\hline 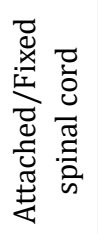 & $-r$ & $r$ & $r$ & $-r$ & - & -1 & - & -1 & -1 & - & $r$ & - & $-r$ & $r$ & $r$ & - & - & -1 & L? \\
\hline 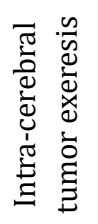 & $r$ & -1 & $r$ & -1 & -1 & -1 & - & - & $\neg$ & -1 & $\rightarrow$ & - & $r$ & - & $r$ & - & $\rightarrow$ & -1 & ?ִ \\
\hline
\end{tabular}




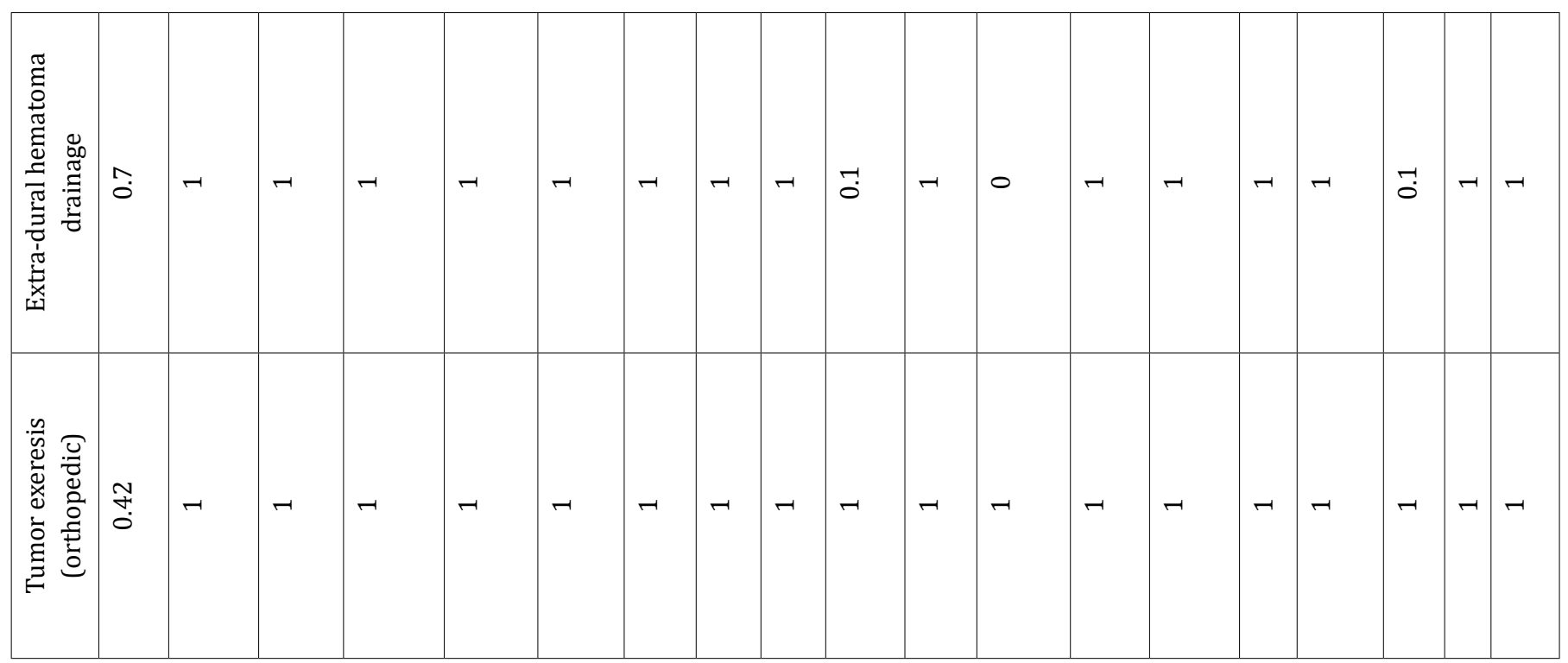

Table 5: p-values Fischer exact test outcomes per surgery.

common cause of multi-organ failure is sepsis with an overall rate of 17 to $73 \%$ [9].

The most common postoperative infection in our study was septicemia (7.22\%), followed by pulmonary (6.19\%) and abdominal sepsis (6.19\%). Postoperative septicemia rates has been reported to vary from 25 to $33 \%$ in liver transplantation surgery $[7,10]$. One study in liver transplantation, reported a postoperative abdominal sepsis of $25 \%$ [10]. In a study in infants aged less than 6 months overall postoperative sepsis was $6.9 \%$ [11]. In this study same, factors related to postoperative sepsis were laparotomy, thoracotomy, diaphragmatic repair, low age and a long intervention time. Independent predictors of postoperative sepsis were central venous catheter and perioperative antibiotics [11].

In-hospital mortality rate was $5.16 \%$ in our study. All patients had a ASA score of more than 3 , were managed on an emergency basis. All the deceased patients had postoperative multi-organ failure and sepsis, all were aged less than 10 months and had co-morbidities. In the literature, mortality rates vary form $0 \%$ in surgery like craniosynostosis to high rates of $17.7 \%$ in liver transplantation, of $19.6 \%$ in ruptured cerebral aneurysms and more than $80 \%$ in patients with multi-organ failure with sepsis [2-6,8-10,12-22].

This study has shown that in children under one year old in major hemorrhagic non cardiac surgery, hemorrhagic shock was the most common intra-operative complication. In our Hospital we do not have transfusion protocols guided with point of care tests [23]. It is time to consider the integration of these protocols in major hemorrhagic surgery to reduce transfusion and improve outcome [23].

One third of patients in this cohort presented intra-operative and or postoperative complications and patients with fatal outcome were all ASA 3 or more. It is time to reconsider the integration of fluid and hemodynamic goal directed therapy in these patients with the aim to improve postoperative evolution since these protocols are not yet a routine practice in our Hospital [24-31].

Postoperative outcome is multifactorial, intra-operative management plays a major role on postoperative evolution. Intra-operative fluid and hemodynamic optimization is one of the keys to upgrade postoperative outcome.

\section{Conclusion}

This secondary analysis of 97 non pre-term infants aged less than one year old, revealed that hemorrhagic shock was the most common intra-operative complication, in the postoperative period, the respiratory system was the most affected system, septicemia was the most common postoperative infection and in-hospital mortality rate was $5.16 \%$. Patients with fatal outcome had ASA scores of 3 or more with severe co-morbidities. 
It is time to reconsider integrating goal directed therapies in intraoperative patient management to improve postoperative outcome.

\section{Conflicts of Interest}

The author declared no conflicts of interest.

\section{Bibliography}

1. Kumba C., et al. "Transfusion and Morbi-Mortality Factors: An Observational Descriptive Retrospective Pediatric Cohort Study". Journal of Anesthesia and Critical Care: Open Access 8.4 (2017): 00315.

2. Beethe AB., et al. "The Road to Transfusion-Free Craniosynostosis Repair in Children Less Than 24 Months Old: A Quality Improvement Initiative". Pediatrics Quality Safety 4 (2020): e331.

3. Bartz-Kurycki M., et al. "Impact of Cardiac Risk Factors on Complications following Cranial Valut Remodeling: Analysis of the 2012-2016 NSQIP-P database". The Journal of Craniofacial Surgery 30.2 (2019): 442-447.

4. Jin SJ., et al. "Risk Factor For Intra-operative massive transfusion in pediatric liver transplantation: a multivariate analysis". International Journal of Medical Sciences 14.2 (2017) :173180.

5. Disma N., et al. "Morbidity and mortality after anaesthesia in early life: results of the European prospective multicentre observational study, neonate and children audit of anaesthesia practice in Europe (NECTARINE)". British Journal of Anaesthesia (2021).

6. Bruce WJ., et al. "Age at Time of Craniosynostosis Repair Predicts Increased Complication Rate". The Cleft Palate-Craniofacial Journal 55.5 (2018): 649-654.

7. Shoji K., et al. "Risk Factors For Bloodstream Infection After Living-donor Liver Transplantation in Children". The Pediatric Infectious Disease Journal 34.10 (2015): 1063-1068.

8. Sinha CK., et al. "The need for Paediatric Emergency Laparotomy Audit (PELA) in the UK". Annals of the Royal College of Surgeons of England 102 (2020): 209-213.
9. Watson RS., et al. "Epidemiology and Outcomes of Pediatric Multiple Organ Dyfunction Sydrome (MODS)". Pediatric Critical Care Medicine 18.3-1 (2017): S4-S16.

10. Kim JE., et al. "Infections after Donor Liver Transplantation in Children". Journal of Korean Medical Science 25 (2010): 527531.

11. Kessler U., et al. "Postoperative Sepsis in Infants Below 6 months of age". World Journal of Pediatrics 5.2 (2009): 113117.

12. Hetts SW., et al. "Intracranial Aneurysms in Childhood: 27Year Single-Institution Experience". American Journal of Neuroradiology 30.7 (2009): 1315-1324.

13. Amelot A., et al. "Long-term Outcomes of Cerebral Aneurysms in Children". Pediatrics 143.6 (2019): e20183836.

14. Binder H., et al. "Management and outcome of traumatic epidural hematoma in 41 infants and children from a single center". Orthopedics and Traumatology: Surgery and Research 102 (2016): 769-774.

15. Nath PC., et al. "Supratentorial extradural hematoma in children: An Institutional Clinical Experience of 65 cases". Journal of Pediatric Neurosciences 10.2 (2015): 114-118.

16. Copeland AE., et al. "Clinical Significance of Venous Anomalies in Syndromic Craniosynostosis". Plastic and Reconstructive Surgery-Global Open 6 (2018): e1613.

17. Haberal M., et al. "Liver Transplantation in Children Weighing Less Than 10 Kilograms". Transplantation Proceeding 38 (2006): 3585-3587.

18. Mack CL., et al. "Living Donor Liver Transplantation for Children With Liver Failure and Concurrent Multiple Organ System Failure". Liver Transplantation 7 (2001): 890-895.

19. Jain A., et al. "Pediatric Liver Transplantation: A single Center Experience Spanning 20 Years". Transplantation 73.6 (2002): 941-947.

20. Beath SV., et al. "Successful Liver Transplantation in Babies Under 1 year”. British Medical Journal 307 (1993): 825-828. 
21. Horsch S., et al. "Volvulus in Term and Pre-term Infants-Clinical Presentation and Outcome". Acta Paeditarica 105 (2016): 623-627.

22. Maas C., et al. "Late-Onset Volvulus Without Malrotation in Extremely Pre-Term Infants- A Case Control Study". BMC Pediatrics 14 (2014): 287.

23. Kumba C., et al. "A Systematic Review and Meta-analysis of Goal Directed Intra-Operative Transfusion Protocols Guided by Viscoelastic Methods and Perioperative Outcomes in Children". International Journal of Recent Scientific Research 10.03 (2019): 31466-31471.

24. Kumba C. "Physiology Principles Underlying Goal Directed Therapies in Children". Research Pediatrics and Neonatology 4.4 ().

25. Kumba C. "Rationale of Goal Directed Therapies in Children". Advances in Pediatric Research 7 (2020): 42.

26. Kumba C. "Do Goal Directed Therapies Improve Postoperative Outcome in Children? (Perioperative Goal Directed Fluid and Hemodynamic Therapy; Transfusion goal directed therapy using viscoelastic methods and enhanced recovery after surgery and Postoperative outcome): A Study Research Protocol". Acta Scientific Paediatrics 2.7 (2019): 17-19.

27. Kumba C. "Goal directed fluid and hemodynamic therapy and postoperative outcomes in children: Value of transthoracic echocardiographic aortic blood flow peak velocity variation: A multi-centre randomized controlled trial protocol". Advances in Pediatric Research 7 (2020): 35.

28. Kumba C. "Trans-Thoracic Echocardiographic Aortic Blood Flow Peak Velocity Variation, Distance Minute, Aortic Velocity Time Integral and Postoperative Outcome in Pediatric Surgical Patients-An Observational Pilot Study Protocol". Open Journal of Internal Medicine 10 (2020): 90-95.

29. Kumba C., et al. "A Systematic Review and Meta- Analysis of Intraoperative Goal Directed Fluid and Haemodynamic Therapy in Children and Postoperative Outcome". Journal of Emergency and Critical Care Medicine 5.1 (2019): 1-9.

30. Kumba C., et al. "Rapid Recovery Pathways after Surgery in Children: A Systematic Review and Meta-Analysis". Medical
Journal of Clinical trials and Case Studies 3.3 (2019): 000211.

31. Kumba C and Melot C. "The Era of Goal Directed Therapies in Paediatric Anaesthesia and Critical Care". EC Emergency Medicine and Critical Care 3.5 (2019): 306-309.

Volume 4 Issue 8 August 2021

(C) All rights are reserved by Claudine Kumba., et al. 